\title{
Astrometric Properties of Gravitational Binary-Microlens Events and Their Applications
}

\author{
Cheongho Han \\ Department of Astronomy \& Space Science, \\ Chungbuk National University, Chongju, Korea 361-763 \\ cheongho@astronomy.chungbuk.ac.kr, \\ Mun-Suk Chun \\ Department of Astronomy, \\ Yonsei University, Seoul, Korea 120-749 \\ mschun@galaxy.yonsei.ac.kr, \\ $\&$ \\ Kyongae Chang \\ Department of Physics, \\ Chongju University, Chongju, Korea 360-764 \\ kchang@alpha94.chongju.ac.kr
}

Received __; accepted _ 


\begin{abstract}
In this paper, we study the astrometric properties of gravitational microlensing events caused by binary lenses. By investigating the centroid shifts for various types of binary-lens events, we find that the deviations of the centroid shift trajectories from the elliptical ones of single-lens events are characterized by distortions, twistings, and big jumps. We study the conditions of binary-lens system configurations and source star trajectories for individual types of deviations. We find dramatic differences in the astrometric centroid shifts for binary-lens microlensing events that would be degenerate had their parameters been determined photometrically. Therefore, when additional astrometric observations of a binary-lens event are available, one can resolve the ambiguity of the binary-lens fit, and uniquely determine the binary-lens parameters.
\end{abstract}

Subject headings: binaries: general - gravitational lensing - astrometry

submitted to The Astrophysical Journal: Apr 13, 1999

Preprint: CNU-A\&SS-04/99 


\section{Introduction}

Searches for gravitational microlensing events that monitor millions of stars located in the Galactic bulge and Large or Small Magellanic Clouds are being carried out by several groups (MACHO: Alcock et al. 1997; EROS: Ansari et al. 1996; OGLE: Udalski et al. 1997; DUO: Alard \& Guibert 1997). Through their efforts more than 300 candidate events have been detected.

The light curve of a single-lens event is represented by

$$
A=\frac{u^{2}+2}{u\left(u^{2}+4\right)^{1 / 2}} ; \quad u=\left[\left(\frac{t-t_{0}}{t_{\mathrm{E}}}\right)^{2}+\beta^{2}\right]^{1 / 2}
$$

where $u$ is the lens-source separation in units of the Einstein ring radius $r_{\mathrm{E}}, t_{\mathrm{E}}$ is the Einstein ring radius crossing time (the Einstein time scale), $\beta$ is the impact parameter, and $t_{0}$ is the time of maximum amplification. The lensing parameters $\left(t_{\mathrm{E}}, t_{0}, \beta\right)$ of the event are obtained by fitting the observed light curve to the theoretical curves given by equation (1.1). Among these parameters, the Einstein time scale provides information about the lens because it is related to the physical lens parameters by

$$
t_{\mathrm{E}}=\frac{r_{\mathrm{E}}}{v} ; \quad r_{\mathrm{E}}=\left(\frac{4 G M}{c^{2}} \frac{D_{o l} D_{l s}}{D_{o s}}\right)^{1 / 2},
$$

where $v$ is the lens-source transverse motion, $M$ is the lens mass, and $D_{o l}, D_{l s}$, and $D_{o s}$ are the separations between the observer, lens, and source star.

On the other hand, when the lens is binary, the light curve deviates from that of the single-lens event in equation (1.1). The most distinctive feature of binary-lens event light curve occurs as a source star crosses a lens caustic (see $\S 2$ ). Whenever a source star crosses a caustic, an extra pair of micro images appears (or disappears), producing a sharp spike in the light curve, giving rise to "strong" binary-lens events. The optimal condition for a strong binary-lens event is that the separation between the two binary components is comparable to the angular Einstein ring radius $\theta_{\mathrm{E}}=r_{\mathrm{E}} / D_{o l}$, corresponding to the combined mass of the binary (combined angular Einstein ring radius). On the other hand, when the binary separation is too small or too large, the event has little chance to involve caustic crossings, resulting in a light curve with a relatively small deviation from that of a single-lens event (i.e. a "weak" binary-lens event; Mao \& Paczyński 1991). Currently a total of 13 candidate binary-lens events have been reported. These include MACHO LMC\#1 (Dominik \& Hirshfeld 1994, 1996; Rhie \& Bennett 1996), OGLE\#6 (Mao \& Di Stefano 1995), OGLE\#7 (Udalski et al. 1994), DUO\#2 (Alard, Mao, \& Guibert 1995), MACHO\#9 (Bennett et al. 1996), 95-BLG-12, 96-BLG-3, 97-BLG-1, 97-BLG-28, 97-BLG-41, 98-BLG-12, 98-BLG-42, and 98-SMC-1 (http://darkstar.astro.washington.edu).

Detecting binary-lens events is important due to their astronomical applications. First, one can obtain physical information about binaries such as mass ratios, $q$, and projected separations, $\ell$. Second, a strong binary-lens event provides an opportunity to measure how 
long it takes for the caustic line to transit the face of the source star. By using the measured caustic crossing time along with an independent determination of the source star size, one can measure the lens proper motion relative to the observer-source line-of-sight, and thus can estimate the location of the lens (Gould 1994; Nemiroff \& Wickramasinghe 1994; Witt \& Mao 1994; Peng 1997). Caustic-crossing events can also be used to determine the radial surface brightness profiles of source stars (Albrow et al. 1999a). Finally, detection of fine structure in the light curve is an excellent method to discover extra-solar planetary systems (Mao \& Paczyński 1991; Gould \& Loeb 1992; Griest \& Safizadeh 1998).

To extract useful information from binary-lens events, it is essential to precisely determine the binary-lens parameters (see $\S 2.1$ ). However, the surface of $\chi^{2}$ behaves in a very complicated manner over the multi-parameter space (Albrow et al. 1999b). As a result, multiple local minima exist, causing ambiguity in the binary-lens fit.

Recently, routine astrometric followup observations of microlensing events with high precision instruments such as the Space Interferometry Mission (hereafter SIM, http://sim.jpl.nasa.gov) have been discussed as a method to measure the distance and mass of MACHOs (Høg, Novikov, \& Polnarev 1995; Paczyński 1998; Boden, Shao, \& Van Buren 1998; Han \& Chang 1999). When a microlensing event is due to a single-point lens, the observed source star image is split into two, and the location of the center of light (centroid) between the two separate images with respect to the position of the source star traces out an ellipse (Walker 1995; Jeong, Han, \& Park 1999). However, if the lens is a binary, both the number and locations of the images differ from those of a single-lens event, resulting in a centroid shift trajectory that deviates from an ellipse.

In this paper, we study the astrometric properties of gravitational microlensing events caused by binary lenses. By investigating the centroid shifts for various types of binary-lens events, we find that the deviations of the centroid shift trajectories from the ellipses of single-lens events are characterized by distortions, twistings, and big jumps. We study the conditions of binary-lens system configurations and source star trajectories for individual types of deviations. We also find dramatic differences in the astrometric centroid shifts for binary-lens events that would be degenerate had their parameters been determined photometrically. Therefore, when astrometric observations of binary-lens events are available in addition to a light curve, one can resolve the ambiguity of the binary-lens fit, and thus determine the binary-lens parameters uniquely.

\section{Binary-lens Events}

When lengths are normalized to the combined Einstein ring radius, the lens equation of a binary-lens event in complex notation is given by

$$
\zeta=z+\frac{m_{1}}{\bar{z}_{1}-\bar{z}}+\frac{m_{2}}{\bar{z}_{2}-\bar{z}},
$$


where $m_{1}$ and $m_{2}$ are the mass fractions of individual lenses, $z_{1}$ and $z_{2}$ are the positions of the lenses, $\zeta=\xi+i \eta$ and $z=x+i y$ are the positions of the source and images, and $\bar{z}$ denotes the complex conjugate of $z$ (Witt 1990). The amplification of each image, $A_{i}$, is given by the Jacobian of the transformation (2.1) evaluated at the image's position, i.e.,

$$
A_{i}=\left(\frac{1}{|\operatorname{det} J|}\right)_{z=z_{i}} ; \quad \operatorname{det} J=1-\frac{\partial \zeta}{\partial \bar{z}} \frac{\overline{\partial \zeta}}{\partial \bar{z}} .
$$

The images and source positions with infinite amplifications, i.e. det $J=0$, form closed curves, called critical curves and caustics, respectively. The total amplification of a source star is given by the sum of the individual amplifications, i.e. $A=\sum_{i} A_{i}$, with $A_{i}$ given by equation (2.2). The position of the source star image centroid is the amplification-weighted average of the positions of individual images, i.e. $\left(x_{c}, y_{c}\right)=\left(\sum_{i} A_{i} x_{i} / A, \sum_{i} A_{i} y_{i} / A\right)$, each of which is obtained by solving the lens equation (2.1) numerically.

\subsection{Light Curves}

To characterize a single-band light curve of a binary-lens event, at least seven parameters are required. These include the binary separation $\ell$ and the mass ratio $q$, the closest separation between the source and the center of the mass of the binary system $\beta$ (impact parameter of a binary-lens event), the time of the closest approach to the center of mass $t_{0}$, the angle that the source star trajectory makes with the projected binary axis $\alpha$ (approaching angle), the combined Einstein ring radius crossing time scale $t_{\mathrm{E}}$, and finally, the baseline source star flux $F_{0}$. In actual experiments in which observations are performed toward very dense star fields one should include an additional parameter for the background flux $B$ to account for blending.

Due to the combination of a large number of binary-lens parameters and the complicated behavior of the $\chi^{2}$ surface over parameter space, fitting the light curve of a binary-lens event is a formidable task (Albrow et al. 1999b). The most serious problem is that there can be multiple local minima, but verifying that one of the local minima is a global solution is very difficult. The ambiguity in binary-lens fits is demonstrated well by Dominik (1999). He showed that several models having a large variety of parameters agree well with the observed binary-lens events OGLE\#7 and DUO\#2. In Figure 1, we reproduce four different model light curves based on the solutions to the binary-lens parameters determined by Dominik (1999) and the measured $I$-band data points of the binary-lens event OGLE\#7 (Udalski et al. 1994). In Figure 2, we present the configurations of the binary-lens system and the source star trajectories for the individual model fits corresponding to the model light curves in Figure 1. In Figure 2, the projected locations of the lenses are marked by ' $x$ ' and the caustics and source star trajectories are represented by the thick curves and thin straight lines respectively. In each panel of Figure 2, we also mark the values of the binary-lens parameters $\ell, q$, and $t_{\mathrm{E}}$. One finds that despite the similarity of the light curves, the resulting binary-lens parameters for individual model fits 
are significantly different from each other.

\subsection{Centroid Shifts of Source Star Images}

When a source star is microlensed by a single point-mass lens, its image is split into two. The typical separation between the two images for a Galactic bulge event caused by a typical stellar mass lens is on the order of a milliarcsecond, and thus one cannot resolve the individual images. However, the source star image centroid shifts caused by microlensing can be measured with high-precision interferometers such as the SIM that will have a positional accuracy of $\lesssim 10 \mu$ arcsec. The centroid shift for a single-lens event is related to the lensing parameters by

$$
\overrightarrow{\delta \theta}_{c}=\frac{\theta_{\mathrm{E}}}{u^{2}+2}\left[\left(\frac{t-t_{0}}{t_{\mathrm{E}}}\right) \hat{\mathbf{x}}+\beta \hat{\mathbf{y}}\right] ; \quad \theta_{\mathrm{E}}=\left(\frac{4 G M}{c^{2}} \frac{D_{l s}}{D_{o l} D_{o s}}\right)^{1 / 2}
$$

where $\theta_{\mathrm{E}}$ is the angular Einstein ring radius, the $x$ and $y$ axes represent the directions which are parallel and normal to the lens-source transverse motion and $\hat{\mathbf{x}}$ and $\hat{\mathbf{y}}$ are the unit vectors for these corresponding directions. If we let $x=\delta \theta_{c, x}$ and $y=\delta \theta_{c, y}-b$, where $b=\beta / 2\left(\beta^{2}+2\right) \theta_{\mathrm{E}}$, the coordinates are related by

$$
x^{2}+\frac{y^{2}}{\mathcal{Q}^{2}}=a^{2},
$$

where $a=\theta_{\mathrm{E}} / 2\left(\beta^{2}+2\right)^{1 / 2}$ and $\mathcal{Q}=b / a=\beta /\left(\beta^{2}+2\right)^{1 / 2}$. Therefore, the trajectory of the apparent source star image centroids traces out an ellipse during the event, known as the 'astrometric ellipse' (Walker 1995; Jeong et al. 1999).

If a lens is binary, on the other hand, the number and positions of the source star images differ from those of a single-lens event(Safizadeh, Dalal, \& Griest 1998). As a result, the centroid shift trajectory of the binary lens event deviates from an ellipse. To see the pattern of the deviations, we compute the centroid shifts for events caused by binaries with various separations and axis ratios and show their trajectories in Figure 3. In Figure 4, we also present the lens system configurations and source star trajectories which are responsible for the centroid shifts in Figure 3. In Figure 4, the caustics and positions of the lenses are represented in the same manner as in Figure 2 and the source star trajectories are represented by straight lines with line types chosen to match those of the centroid shift trajectories in Figure 3. The impact parameters of the individual source star trajectories are $\beta=0.3$ (solid lines), 0.5 (dotted lines), and 0.7 (dashed lines), and the approaching angle $\alpha$ is $20^{\circ}$ for all trajectories.

From Figure 3, one finds that the diversity of the centroid shift trajectories expected from binary-lens events is very large, similar to photometric light curves. However, despite the large variations, one finds that the deviations of the centroid shift trajectories from the astrometric ellipses of single-lens events can be classified by having distortions, 
twistings, and big jumps. First, distortions in the trajectories of centroid shifts occur for non-caustic-crossing binary-lens events in which binary separations are relatively small compared to their impact parameters. For an event with a very close binary separation, i.e. $\ell \ll \beta$, the centroid shift trajectory is well approximated by the astrometric ellipse of a single-lens event with mass equal to the total mass of the binary and located at the center of mass of the binary. However, we note that for the same binary-lens event the fractional deviation of the astrometric centroid shifts from those of the corresponding single-lens event is significantly larger than the fractional deviation of the photometric amplifications, making astrometrically observing microlensing events a useful method to detect very close binaries (Chang \& Han 1999). The amount of deviation increases with an increasing ratio of $\ell / \beta$. Secondly, when the binary separation is equivalent to the impact parameter, i.e. $\ell \sim \beta$, the trajectory of the centroid shift becomes twisted, developing a loop near the closest approach. For a given lens system configuration, the size of the loop is bigger for events with smaller impact parameters. We note that similar distortions and twistings occur due to the effect of finite source size (Mao \& Witt 1998). However, while the source star trajectories deviated by the finite source effect are symmetric with respect to the line connecting the position of the unlensed source star and the centroid at the time of maximum amplification, the centroid shift trajectories caused by binary lenses are, in general, asymmetric. Therefore, one can distinguish the trajectories of astrometric centroid shifts caused by binary lenses from those affected by the finite source effect. Finally, whenever the source star crosses a caustic, the centroid of the source star images shifts by a large amount, up to about 0."001 - 0."01 (Witt \& Mao 1996), producing a big jump in the centroid shift trajectory.

\subsection{Application of Astrometric Observations}

In the previous subsection, we showed that, depending on the lens-system configurations and source star trajectories, i.e. binary-lens parameters, the trajectories of the centroid shifts of binary-lens events take various forms. Thus, one can determine the binary-lens parameters from the centroid shift trajectory in a manner similar to the determination of parameters from the light curve. To fit a centroid shift trajectory, however, the number of parameters is almost the same as the number to fit a light curve. I The result is

\footnotetext{
${ }^{1}$ For an actual observation of a microlensing event, there are two more parameters needed to describe the centroid shift than are needed to describe the light curve. These parameters are used to represent the position of the blended star $\left(x_{b}, y_{b}\right)$ because the centroid shifts are not only affected by the flux of the blended star, but also by its location. However, one can correct for the effect of nearly all types of blending, and thus both the values of $\left(x_{b}, y_{b}\right)$ and $B$ can be determined by using the diffraction-limited resolution of space-borne observations and the high positional accuracy of the SIM (Han \& Kim 1999). Therefore, the effective number of parameters necessary to describe the centroid shift trajectory is one less than the
} 
that the astrometric determination of the binary-lens parameters suffers from the same degree of ambiguity as the photometric determination. Therefore, there is no advantage to determining the binary-lens parameters from the centroid shifts alone.

However, one can completely resolve the ambiguity in finding the parameters by using both the photometric light curve and the astrometric centroid shift trajectory. This is because the astrometric centroid shifts expected from the photometrically determined binary-lens parameters are significantly different from each other. To demonstrate this, in Figure 5 we present the trajectories of the centroid shifts that are expected for the four degenerate lens-parameter solutions corresponding to the model light curves in Figure 1. From Figure 5 one finds that despite the very similar the light curves, the trajectories of the centroid shifts differ dramatically from each other. To isolate a global solution, the measured centroid shift trajectory is simply compared to the solutions producing the photometric fit.

\section{Summary}

The findings from our investigation of the astrometric properties of binary-lens gravitational microlensing events are summarized as follows.

1. The trajectories of centroid shifts expected from binary-lens events take various forms depending on the lens-system configurations and source star trajectories.

2. Despite the large diversity of centroid shift trajectories, the deviations from the elliptical trajectories of single-lens events can be categorized into distortions, twistings, and large jumps.

3. The centroid shift trajectories expected from different degenerate solutions of the photometrically determined binary-lens parameters are dramatically different from each other. Therefore, with the additional information provided by the astrometrically determined centroid shifts, one can completely resolve the ambiguity of the photometric binary-lens fit, and thus can uniquely determine the binary-lens parameters.

We would like to thank M. Everett for careful reading of the manuscript. M. S. Chun was supported by the financial grant from the Korea Research Foundation (1998-015-D00287) in the program year of 1999.

number of parameters needed to characterize the light curve. 


\section{REFERENCES}

Alard, C., Mao, S., \& Guibert, J. 1995, A\&A, 300, L17

Alard, C., \& Guibert, J. 1997, A\&A, 326, 1

Albrow, M. D., et al. 1999a, preprint (astro-ph/9903008)

Albrow, M. D., et al. 1999b, ApJ, submitted (astro-ph/9811479)

Alcock, C., et al. 1997, ApJ, 486, 697

Ansari, R., et al. 1996, A\&A, 314, 94

Bennett, D. P., et al. 1996, Nucl. Phys. Proc. Suppl., 51B, 152

Boden, A. F., Shao, M., \& Van Buren, D. 1998, ApJ, 502, 538

Chang, K., \& Han, C. 1999, ApJ, in press

Dominik, M. 1999, A\&A, 341, 943

Dominik, M., \& Hirshfeld, A. C. 1994, A\&A, 289, L31

Dominik, M., \& Hirshfeld, A. C. 1996, A\&A, 313, 841

Gould, A. 1994, ApJ, 421, L71

Gould, A., \& Loeb, A. 1992, ApJ, 396, 104

Griest, K., \& Safizadeh, N. 1998, ApJ, 500, 37

Han, C., \& Chang, K. 1999, MNRAS, 304, 845

Han, C., \& Kim, T.-W. 1999, MNRAS, 305, 795

Høg, E., Novikov, I. D., \& Polnarev, A. G. 1995, A\&A, 294, 287

Jeong, Y., Han, C., \& Park, S.-H. 1999, ApJ, 511, 569

Mao, S., \& Di Stefano, R. 1995, ApJ, 440, 22

Mao, S., \& Paczyński, B. 1991, ApJ, 374, L37

Mao, S., \& Witt, H. J. 1998, MNRAS, 300, 1041

Nemiroff, R. J., \& Wickramasinghe, W. A. D. T. 1994, ApJ, 424, L21

Paczyński, B. 1998, 404, L23

Peng, E. 1997, ApJ, 475, 43

Rhie, S. H., Bennett, D. P. 1996, Nucl. Phys. Proc. Suppl., 51B, 86

Safizadeh, N., Dalal, N., \& Griest, K. 1998, preprint (astro-ph/9811233)

Udalski, A., et al. 1994, ApJ, 436, L103

Udalski, A., et al. 1997, Acta. Astron., 47, 169

Walker, M. A. 1995, ApJ, 453, 37

Witt, H. J., \& Mao, S. 1994, ApJ, 430, 505 
Witt, H. J., \& Mao, S. 1996, in IAU Symp. 173, Astrophysical Applications of Gravitational Lensing, ed. C. Kochanek \& J. Hewitt (Dordrecht: Kluwer), 233 


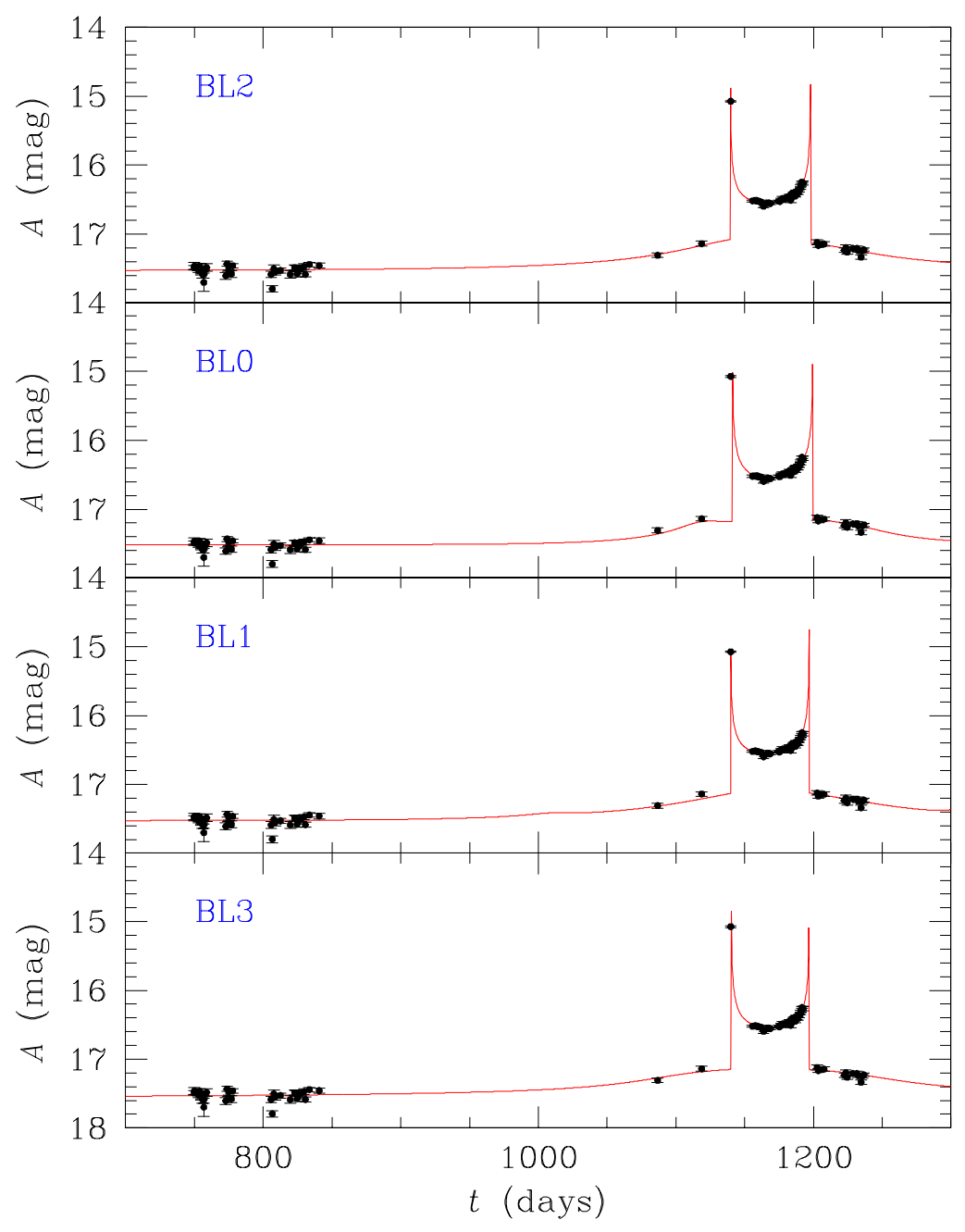

Figure 1: The measured $I$-band data points of the binary-lens event OGLE\#7 and the four different model light curves (solid lines). The designations of individual model light curves (BL2, BL0, BL1, and BL3) are borrowed from Dominik (1999) who originally determined the binary-lens parameters for the model light curves. 


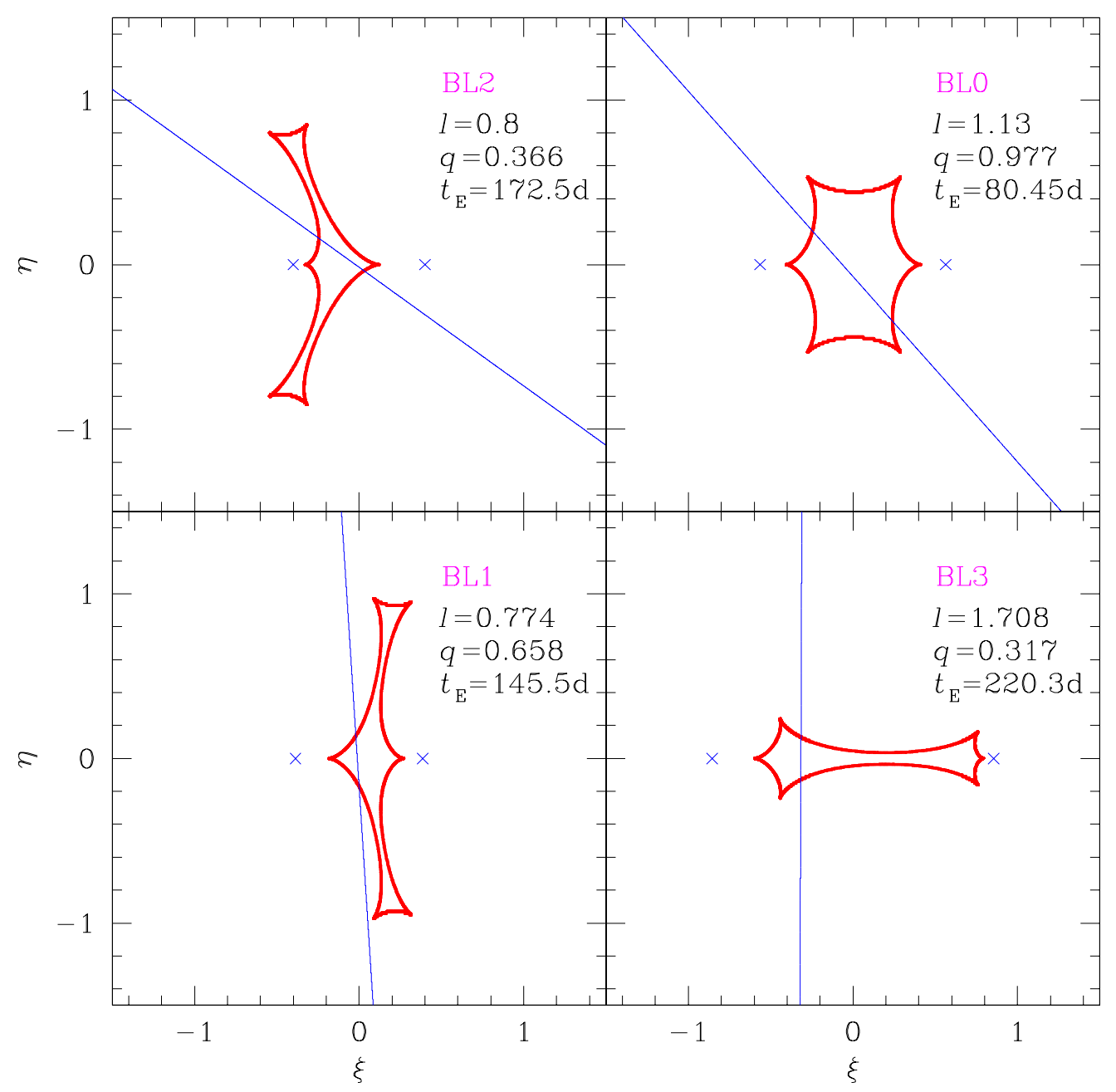

Figure 2: The configurations of the binary-lens systems and source star trajectories (thin solid lines) that correspond to the model light curves in Figure 1. For each configuration, the lens locations and the caustics are marked by ' $x$ ' and thick solid lines respectively. Also marked in each panel are the values of the binary-lens parameters $\ell, q$, and $t_{\mathrm{E}}$. One finds that despite the similarity of the light curves (in Figure 1), the difference in the binary-lens parameters are significant. 


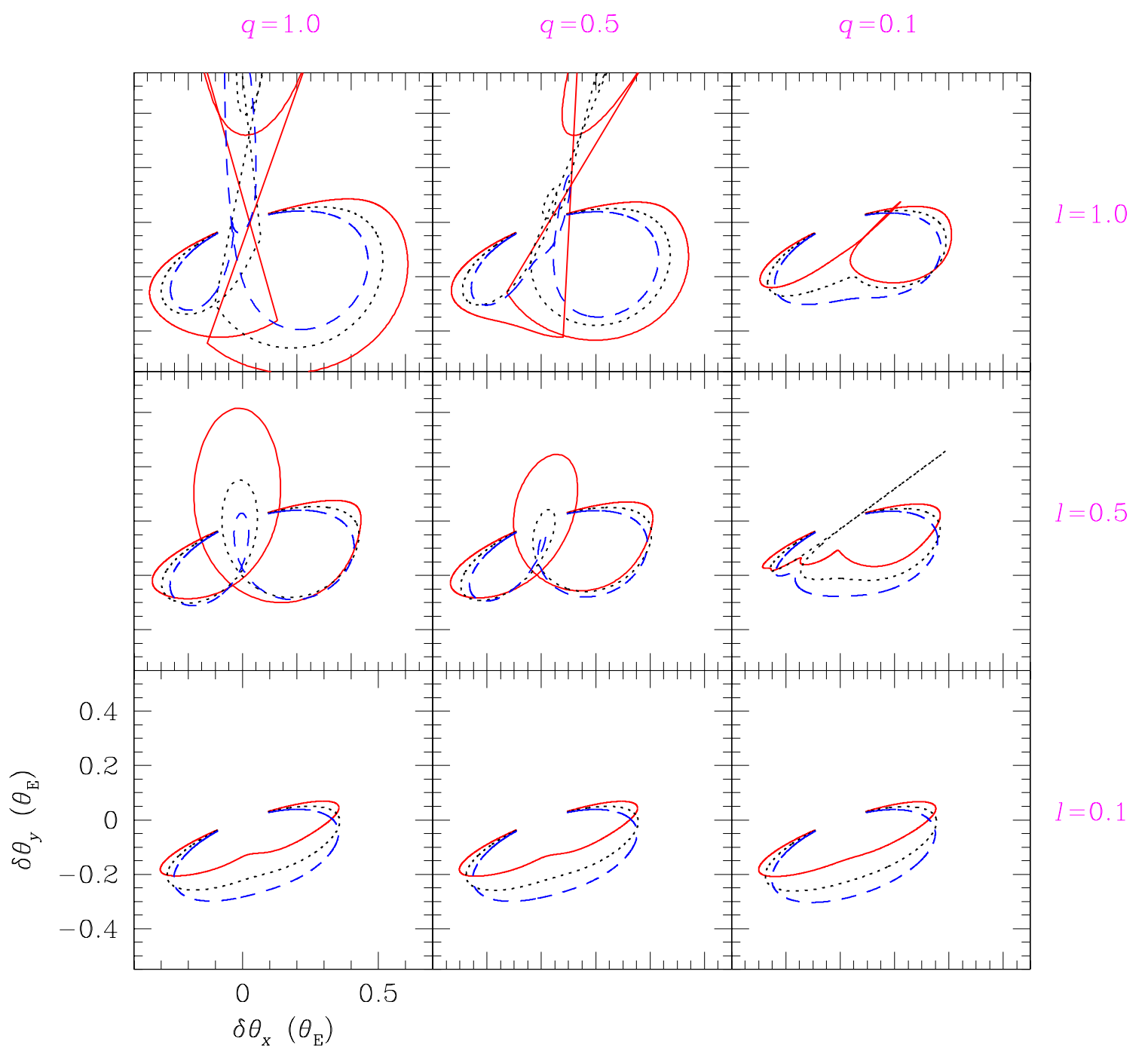

Figure 3: Trajectories of source star image centroid shifts for microlensing events caused by binary lenses with various binary separations, $\ell$, and mass ratios $q$. 


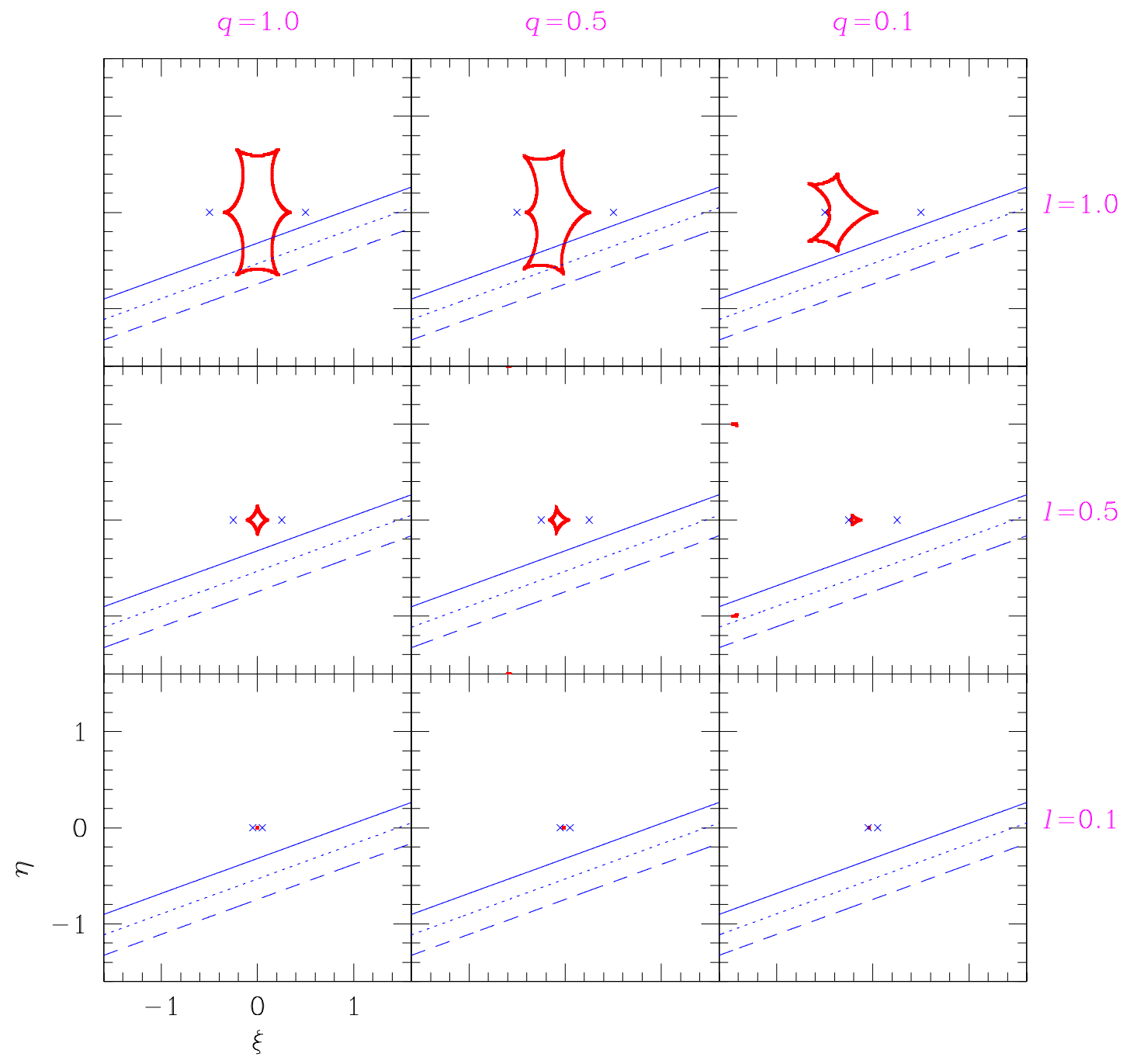

Figure 4: The lens system configurations and source trajectories (thin solid lines) which are responsible for the centroid shift trajectories in Figure 3. The caustics and the positions of lenses are represented in the same way as in Figure 2. The trajectories have the closest separations of $\beta=0.3$ (solid lines), 0.5 (dotted lines), and 0.9 (dashed lines) and have the same approaching angle of $\alpha=20^{\circ}$. The line type of each source star trajectory is chosen so that it matches with the line type of the centroid shift trajectory in Figure 3. 


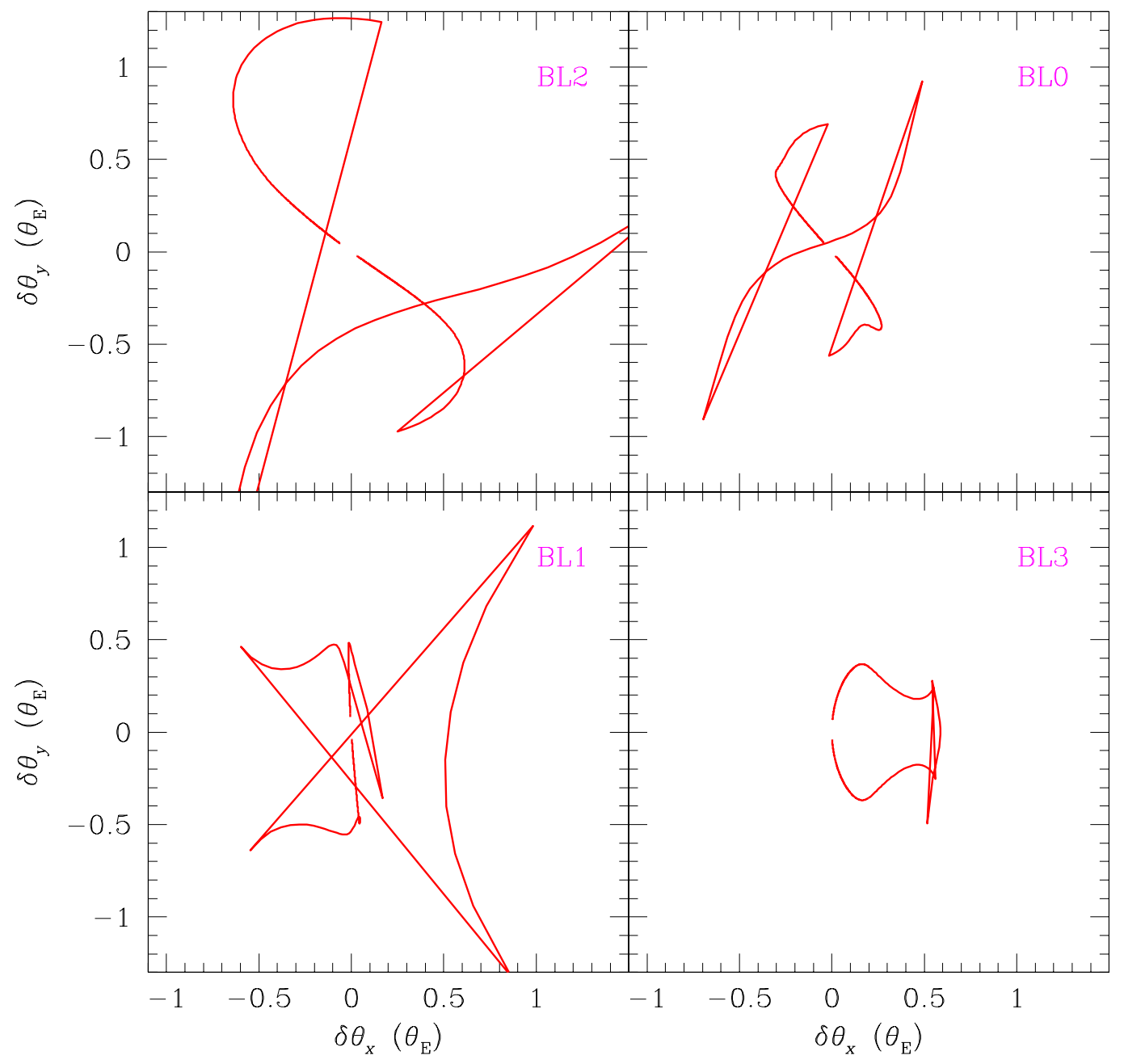

Figure 5: The centroid shift trajectories that are expected for the four lens parameters solutions corresponding to the model light curves in Figure 1. One finds that despite the similarity in the light curves, the centroid shift trajectories are dramatically different from one another. 